Nicholai Studies, Vol. I, No. 1 (2021): 220.

DOI: https://doi.org/10.46825/nicholaistudies/ns.2021.1.1.220

\title{
Ethical and Scientific Standards
}

The journal Nicholai Studies is completely non-profit. The publication of the Nicholai Studies is financed exclusively by donations and subscriptions. The whole process of preparing the journal for publication is completely free for all involved, both for authors and reviewers and the Editorial Board, starting from writing articles and texts for the Nicholai Studies, through receiving articles for the journal, peer reviews, possible subsequent corrections until the end of the preparation for the press and the publication itself.

The Editorial Board of the Nicholai Studies holds to very high ethical and academic standards in the publication of scientific papers.

Therefore, only scientific works that are the result of an independent research by one or more authors, the ones that meet the language and academic standards, including thorough treatment of themes, that use the latest scientific literature, and that respects the academic standards, are going to be published in Nicholai Studies.

\section{Plagiarism}

Authors are required to ensure that their work is fully original, and if someone else's work is used, that it is properly quoted and referenced. Active anti-plagiarism checks shall be carried out, both by the Editorial Board of the Nicholai Studies and by reviewers.

Plagiarism means presenting someone else's work as one’s own, using others' ideas, concepts, words, structures, without proper quoting and without referencing the source. Plagiarism represents literally taking someone's work (copy/paste), in whole or in part, without specifying the origin and without quotation marks. This also applies to articles that are not authorized, such as various internet websites, blogs, and so on. Plagiarism includes the use of other's work without citing sources even when they are not literally transferred but paraphrased or translated from another language and so on.

Except in special cases (analysis of sources etc.), the paper should not contain more than $20 \%$ of the literally quoted text, even when the source is marked. 